\title{
La migración en la construcción de territorios y lugares. El caso de Pedro Luro, Argentina
}

\section{The migration in the construction of territories and places. The case of Pedro Luro, Argentina}

\author{
Marcela Torrez Gallardo \\ Licenciada en Geografía. Becaria Doctoral del Consejo Nacional de Investigaciones Científicas y Técnicas CONICET. Departamento de Geografía \\ y Turismo. Universidad Nacional del Sur. 12 de Octubre y San Juan, $4^{\circ}$ Piso, Gabinete 12, (8000) Bahía Blanca, Buenos Aires, Argentina, \\ torrez_marcela@hotmail.com
}

Recibido: 1 de noviembre 2019 || Aprobado: 22 de abril 2020

\section{Resumen}

El presente trabajo introduce el análisis de un barrio y la feria regional de la localidad de Pedro Luro, al sudoeste de la provincia de Buenos Aires (Argentina), desde una reflexión teórica empírica; centrada en categorías tales como territorio, lugar e identidad. Desde el abordaje cultural de la Geografía, se pretende analizar las prácticas socio-espaciales que efectúan los migrantes como sujetos situados que construyen territorios y lugares. Pedro Luro, como estudio de caso, tiene una trayectoria histórica-territorial ligada a la migración de tipo europea en sus inicios y posteriormente de tipo regional-limítrofe. Actualmente el mayor conjunto migrante está constituido por la colectividad boliviana con una interesante impronta cultural en lo territorial, social y económico. La investigación tiene un carácter cualitativo, cuya metodología está fundamentalmente apoyada en la observación participante, las entrevistas semi-estructuradas y el análisis de las representaciones.

Palabras clave: Migrantes; Lugares; Territorio; Identidad

\section{Abstract}

The present essay introduces the analysis of a neighborhood and the regional fair of Pedro Luro city, southwest of Buenos Aires province (Argentina), from a theoretical empirical reflection; centered on categories such as territory, place and identity. From the cultural approach in Geography, the aim is to analyze the socio-spatial practices that affect the migrants as placed subjects that construct territories and places. Pedro Luro, as a case study, has a historical-territorial path tied to the European migration in its beginnings and later of a regional-bordering type. Currently the largest migrant group is constituted by the Bolivian collectivity with an interesting cultural stamp in the territorial, social and economic areas. The research has a qualitative character, which methodology is fundamentally supported in participant observation, semi-structured interviews and analysis of representations.

Key words: Migrants; Places; Territory; Identity 


\section{INTRODUCCIÓN}

Históricamente la sociedad argentina se fue constituyendo por la llegada de inmigrantes europeos a fines del siglo XIX y principios del siglo $X X$, fomentada a partir de la imperiosa necesidad de generar el asentamiento de estos flujos poblacionales (Devoto, 2003). Posteriormente se reconoce una inmigración latinoamericana del siglo $X X$ y $X X I$ de tipo más contemporánea, caracterizada por ser originaria de países limítrofes. Indudablemente los contextos institucionales y de derechos en que efectivamente se desplegaron las trayectorias migratorias en estos recortes históricos fueron muy distintos. Actualmente la población inmigrante latinoamericana presente en la Argentina se caracteriza principalmente por provenir de países como: Bolivia, Chile, Paraguay, Uruguay y Perú (Pacceca, 2009; Pizarro, 2011; Benencia, 2007; Halpern, 2009). En un contexto de urbanización regional, las migraciones han sido uno de los factores contribuyentes en el crecimiento y expansión de los espacios urbanos en la Argentina, asociadas a sus posibilidades de inserción laboral. Las ciudades emergen como espacios donde se expresa la materialidad más axiomática de las prácticas sociales ( $\sin$ excluir a los espacios rurales) de muchos grupos migrantes (Sassen, 2006).

Pedro Luro es una pequeña localidad en el partido de Villarino (Figura 1), cuyo crecimiento ha sido significativo en términos comparativos para la región del sudoeste de la provincia de Buenos Aires (Argentina). Según el último Censo Nacional de Población del año 2010, el partido de Villarino registraba 31.014 habitantes, siendo el distrito que más creció en la provincia de Buenos Aires junto con el conurbano, en gran parte debido a la radicación de población migrante. Villarino tiene un $10,7 \%$ de su población nacida en el extranjero, de los cuales 3.135 habitantes provienen de países limítrofes y 2.276 habitantes son nacidos en Bolivia. De las localidades del partido, Pedro Luro es la más poblada con 9.494 habitantes. Del total un $12 \%$ de su población corresponde a la nacionalidad boliviana según base de datos REDATAM (INDEC, 2010). Con el transcurrir de estos últimos años, se incrementó el número de habitantes tanto en la localidad como en el partido. En la actualidad, según estimaciones del INDEC (2015) y las realizadas por el Municipio, en Villarino residen aproximadamente 35.000 habitantes, de los cuales el 30\% corresponde a la comunidad boliviana. Mientras que en Pedro Luro, se estima que su población actual es de 15.000 habitantes, con un 30\% representado por la colectividad boliviana.

El presente trabajo pretende evidenciar a partir de un estudio de caso, cómo los migrantes re-construyen territorios y lugares a partir sus prácticas socio-espaciales. A los fines del presente trabajo, solo se tomará como espacio de análisis la experiencia empírica sobre un barrio de la ciudad, donde se visibilizan las territorialidades de los migrantes bolivianos, como uno de los grupos más dominantes. Realizar estudios e investigaciones sociales posee una complejidad no solo empírica por tratarse de sujetos migrantes sino, por la amplitud y variedad de posibles enfoques epistemológicos, teóricos y metodológicos del tema. En este sentido se desarrollará un primer apartado con los lineamientos teórico-conceptuales, que posibilitarán vislumbrar la problemática priorizando el abordaje cultural desde la disciplina geográfica y haciendo foco sobre categorías como territorio, espacio, lugar y territorialidad. También se hará referencia a la cuestión de las migraciones, haciendo algunas salvedades teórico-conceptuales para contextualizar el caso de análisis y el enfoque metodológico de base cualitativa sobre la cual se fundamenta la presente investigación. Posteriormente se hará hincapié en el análisis concreto de cómo los migrantes re-construyen territorios y lugares en la ciudad de Pedro Luro a través de las prácticas socio-espaciales $y$, finalmente, se expondrán las aproximaciones reflexivas de la presente investigación. 
Figura 1. Ubicación de Pedro Luro en el sudoeste de la provincia de Buenos Aires (Argentina)
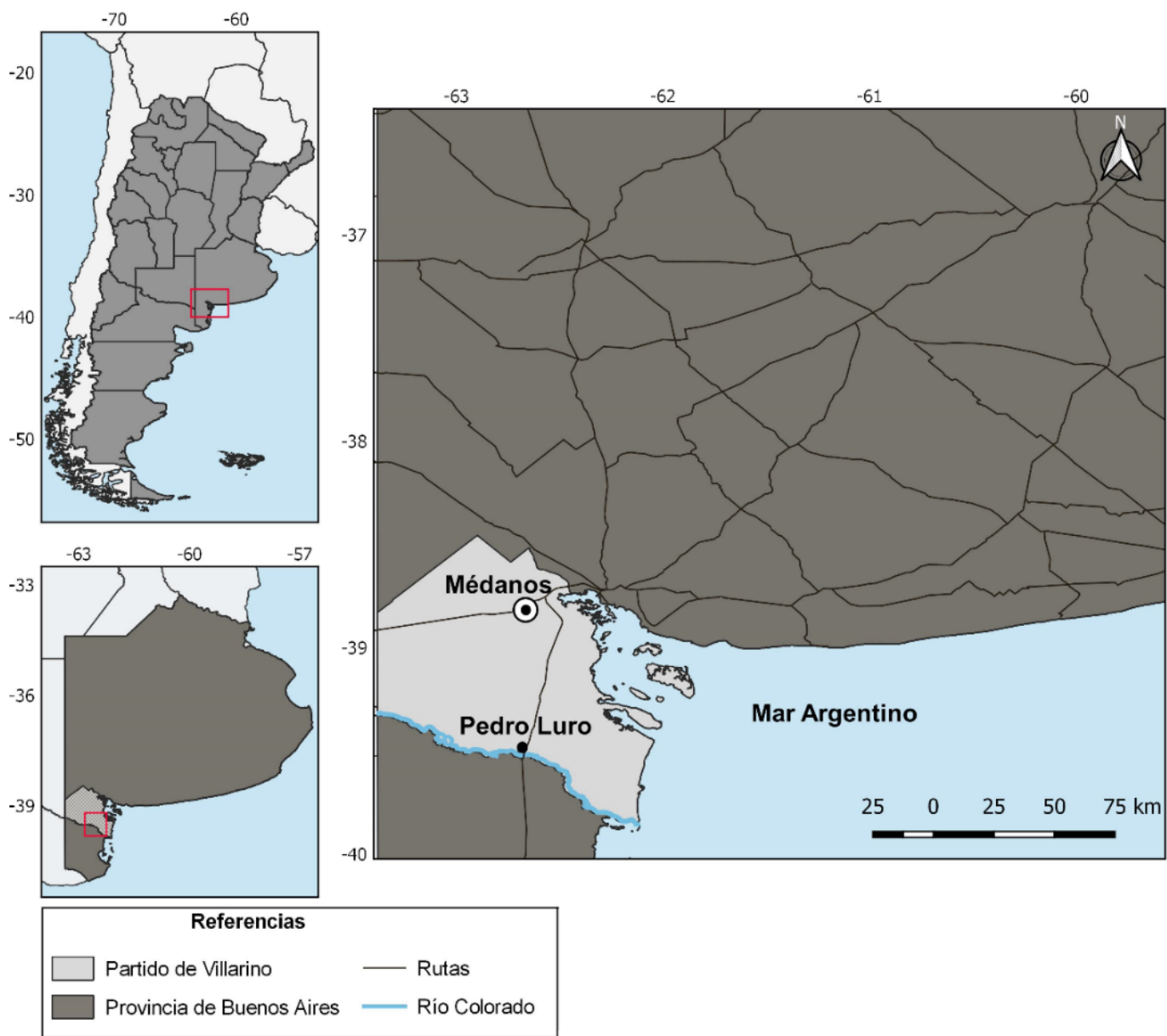

Fuente: elaborado por Buzzi con base en información provista por el Instituto Geográfico Nacional

\section{Perspectivas teórico-CONCEPTUAles: TERritorio, LUGAR Y MIGRACIONES}

Desde las geografías posmodernas y con la crisis de los paradigmas tradicionales, la ciudad se convierte en un nuevo modelo para los estudios sociales y urbanos (Carreras y García Ballesteros, 2006), enfatizando la visión analítica de la fragmentación de su espacio y por los nuevos conflictos sociales y culturales (Soja, 2000; Scoot y Soja, 1996). En el contexto de los nuevos "giros" de la disciplina, la Geografía Cul-

1 A partir de 1970 se produce un cambio profundo en las concepciones de la geografía, sobre todo en temas ligados al aspecto social conocido como el "giro" cultural en la geografía. Claval (2002) menciona que "el sentido del giro cultural no es solo el de explorar un nuevo campo abierto a la investigación, sino también el de repensar totalmente la disciplina" (p. 294). tura $\left.\right|^{2}$ se interesa en comprender el significado de lo representado por los individuos; el modo en como comprenden y perciben su ambiente; donde la cultura aparece como el medio para estudiar las colectividades de individuos y; la manera en cómo transforman el mundo material a partir de un mundo de símbolos que otorga sentidos y valoraciones (Fernández Christlieb, 2006). Este último expresa que el objeto de

2 El desarrollo histórico de la Geografía Cultural ha puesto en evidencia los cambios en la manera de estudiar las realidades sociales (Claval, 2002; Wagner, 2002; Clua y Zusman, 2002; Fernández Christlieb, 2006). Desde la perspectiva de qué, se ocupa de estudiar los rasgos visibles de la cultura (Sauer, 1995) hasta entender que esta también se expresa en paisajes simbólicos (Cosgrove, 2003, en Fernández Christlieb, 2006), ha habido no solo cambios de perspectivas sino también cambios en sus metodologías de trabajo. 
estudio central de esta geografía es la dimensión espacial, mediante la definición de sus categorías específicas como: región, territorio, sitio, lugar, paisaje, entre otras. Las especificidades de lugar y el territorio; los cuales están cargados de afectividades, sentimientos y emociones; son entendidas como 'espacios con sentido' (Bustos Cara, 2002). Existen gran variedad de definiciones respecto a territorio y lugar, sin embargo, a los fines prácticos del presente trabajo, y tomando los aportes de Torres (2011), se podría decir que la construcción de territorios obedece a las interacciones y experiencias sociales que permitan su control (son las relaciones sociales de poder y apropiación lo que definen a los territorios), mientras que los lugares responden a su apropiación subjetiva. Estos últimos,

están indisolublemente ligados a los sentimientos de identidad, puesto que algunos sirven como puntos de reunión para los que se sienten próximos. Conservan, en su paisaje, signos que han aprendido a valorar. Los lugares de identidad, cuando son adyacentes, forman conjuntos coalescentes y constituyen territorios. (Claval, 2002, p. 32)

Al respecto, Lindón (2007) (desde una perspectiva constructivista) afirma que los lugares pueden ser construidos a diario desde la perspectiva del propio sujeto habitante y también cognoscente, aunque esos lugares también pueden reconfigurar las identidades de los sujetos que los habitan. En este sentido la autora expresa que:

...cada lugar es resultado de las acciones del sujeto sobre el mundo externo, dependiendo así tanto de las características del sujeto como de las del entorno en el cual se ejerce la acción. A su vez, las acciones del sujeto habitante se configuran dentro de una lógica compartida y también reconstruida constantemente. Al mismo tiempo, esos lugares así construidos modelan esas tramas de sentido y las acciones que en ellos se concretan. (2007, p. 36)

La combinación de ambas categorías es la que permite comprender la espacialidad como dimensión central y constitutiva de las prácticas sociales expresadas por los migrantes en la ciudad de Pedro Luro. Estas prácticas visibilizadas en los lugares de feria ancladas en un territorio barrial de la ciudad, tienen una connotación fuertemente cultural asociada a las comunidades migrantes de origen boliviano que allí habitan. En este sentido, el barrio es entendido como un territorio histórico y socialmente construido, como una categoría constitutiva de la forma de percepción, significación y acción por los propios sujetos. Se define como un espacio social en el que se observan marcas culturales propias de la colectividad. La concentración de migrantes en un barrio es producto de las redes sociales y la necesidad comunitaria de construir lugares (Sassone y Mera, 2007; Sassone y Hughes, 2009). Por ello resulta pertinente hacer una breve referencia a las nociones teóricas que comprenden las migraciones en el contexto de las multidimensionalidades que implican como procesos sociales.

En sociedades tan heterogéneas y culturalmente diversas, grupos sociales como los migrantes pueden expresarse a partir de sus prácticas sociales en territorios diferenciados, con un mayor grado de localización geográfica y reproduciendo sus formas culturales (alimentación, vestido, celebraciones rituales y religiosas) en espacios y momentos específicos. La 'segregación' es un proceso inherente a las sociedades y su aspecto espacial, a través del alejamiento o cercanía de prácticas y representaciones sociales, construye un espacio urbano socialmente diferenciado, el cual, por medio de la heterogeneidad y diversidad, son parte de las características de las ciudades actuales (Garín Contreras, 2013). Por su parte Zygmunt Bauman³ (1994) entiende que no hay un sentimiento de pertenencia sin sentimiento de exclusión y viceversa. Ambos términos adquieren significados a partir de la oposición. Las acciones colectivas de apropiación a lo largo del tiempo generaron que espacios inicialmente segregados se convirtieran

\footnotetext{
3 Nosotros y ellos, los que pertenecemos al grupo y a los foráneos, derivamos nuestras respectivas características, como también nuestros matices emocionales, de nuestro mutuo antagonismo. Podríamos decir que nuestro antagonismo define ambos lados de la oposición. Podríamos decir también que cada lado saca su identidad del hecho mismo de que lo vemos comprometido en un antagonismo con el lado opuesto (Bauman, 1994, p. 45).
} 
en 'aparentes espacios de integración'. Los grupos migrantes establecen una particular forma y estrategia de asentamiento e integración para con la sociedad mayor, y ello se traduce en variados 'tipos de inserción' que afectan a los distintos conjuntos, diferentes modos de relación que desean y/o logran establecer con la sociedad (considerada como totalidad) de la que forman parte (Chmiel, 1999).

Desde una perspectiva teórica, las migraciones pueden ser entendidas más allá que solo simples movilidades ${ }^{4}$, ya que significan un proceso social que "(...) comprende una compleja serie de cambios a nivel individual, familiar y comunitario" (Massey, 2017, p. 63). El mismo autor establece que como proceso, puede ser visto como un conjunto singular e integrado de cambios que actúan juntos produciendo un resultado particular. El estudio de las migraciones en la Argentina es amplio, desde distintos enfoques disciplinares (Grimson y Jelin, 2006; Feldman Bianco, Rivera Sánchez, Stefoni y Villa Martínez, 2011) y desde diferentes dimensiones de análisis (Benencia, 2006; Sassone, 2007; Pacceca, 2009). En la actualidad el desplazamiento de personas es un tema complejo tanto por las diferentes causas que lo motivan, como por las consecuencias que generan, un ejemplo de ello es la construcción de nuevas territorialidades transnacionales (Tarrius, 2009; Cortès, 2009; Lara Flores, 2012). De este modo las migraciones aparecen en el foco de un amplio campo disciplinar, cuya relevancia está centrada no solo en el análisis académico sino en su implicancia para el desarrollo de políticas públicas (Novick, 2008; Pizarro, 2011; Wihtol de Wenden, 2013; Massey, 2017).

Realizar estudios asociados a las migraciones implica la necesidad de poder hacerlo desde un enfoque multidimensional, es decir desde las 'multicausalidades' del proceso (tanto del país de origen como el de destino); 'el carácter

4 La Organización Internacional para las Migraciones (OIM) define a la migración como el "movimiento de población hacia el territorio de otro Estado o dentro del mismo que abarca todo movimiento de personas sea cual fuere su tamaño, su composición o sus causas" (2006, p. 38). multifacético' (como los efectos económicos, la construcción de imaginarios sociales y estereotipos) y el 'anclaje local' (desde la perspectiva de la 'glocalización'). Pero también resulta importante estudiar las migraciones desde 'la subjetividad y el plano identitario' del sujeto (Lamborghini y Martino, 2018). Es en esta dimensión donde aparecen las emociones, sentimientos y afectividades del migrante guardados con su lugar de origen, visibilizadas y proyectadas en su nuevo lugar de destino. $Y$ finalmente aparece el 'transnacionalismo' asociado a las redes de migrantes, que en algunos casos actúan como intermediarios. Dentro de estas relaciones se podrían considerar a las redes familiares transnacionales, que organizan la ubicación residencial y laboral de los migrantes a partir de relaciones familiares y vecinales. También podría ser el caso de los migrantes que conforman los mercados populares y actúan en calidad de productores, vendedores o consumidores de mercancías, garantizando flujos comerciales que algunos investigadores estudian en términos de 'globalización desde abajo' o 'globalización popular' (Lins Ribeiro, 2012). En el análisis de las prácticas espaciales del barrio y de la feria en Pedro Luro, se retoman estas dimensiones del enfoque multidimensional de las migraciones, puesto que resultan necesarias para comprender el contexto en el que se desarrollan estas expresiones espaciales de carga subjetiva; que, si bien tienen una magnitud de escala local, no pueden ser entendidas sin su dimensión relacional a escala regional.

\section{ENFOQUE METODOLÓGICO DE ANÁLISIS}

Para comprender cómo las prácticas socio-espaciales de los migrantes re-construyen territorios y lugares en el área de estudio, fue necesario partir de una investigación de tipo cualitativa, tomando como caso de análisis la ciudad de Pedro Luro y la experiencia empírica sobre un barrio concreto de la misma. Puesto que tal categoría espacial posee la relevancia de expresar claramente las territorialidades de los migrantes de origen boliviano, definidos como 
los grupos migrantes más dominantes de la región para estos últimos años. La investigación cualitativa de acuerdo a Vasilachis de Gialdino (2006) supone la inmersión en la vida cotidiana del objeto de estudio; la valoración y el intento por descubrir la perspectiva de los participantes sobre sus propias realidades y; la consideración de la investigación como un proceso interactivo entre el investigador y esos participantes. En este contexto, la metodología empleada tiene un carácter sociocultural y de tipo participativo. Las técnicas de trabajo ${ }^{5}$ y fuentes de información se apoyaron en las entrevistas semi-estructuradas a informantes clave, la recopilación de las historias de vida de los sujetos migrantes y la observación participante.

Dentro de los informantes clave a quienes se dirigieron las entrevistas, se encuentran: los migrantes tradicionales y recientes (chilenos, bolivianos y paraguayos); la comunidad migrante boliviana (comprendiendo a las generaciones de hijos/as nacidos/as en territorio argentino) y; los no-migrantes (correspondientes a aquellas generaciones de origen mayoritariamente europea, que conformarían la sociedad 'local' o 'criolla'). Por tanto, los criterios que se tomaron en cuenta para la selección de los informantes fueron la trayectoria de permanencia y residencia en Pedro Luro, el país de origen y la diferencia etaria (de allí la importancia de considerar a las generaciones nacidas en Argentina). Cabe aclarar que el foco está puesto en los migrantes bolivianos, pero también interesa entender sus especificidades respecto de otros colectivos, así como el sentido relacional con la población 'local' o criolla. Puesto que la construcción de territorios y lugares de un determinado grupo social, también está determinada por la adscripción que les es atribuida por otros actores o sujetos que forman parte del contexto urbano de Pedro Luro.

En este sentido, las entrevistas también es-

5 Según Scribano (2008) existen cuatro técnicas primarias para llevar adelante una investigación cualitativa, basado en la propuesta de Marshall y Rossman (1995) serían: la participación, la observación, las entrevistas en profundidad y la revisión documental. tuvieron destinadas a los representantes de las instituciones públicas, de organizaciones sociales, educativas entre otros, con la intencionalidad de contar con una mayor diversidad de perspectivas de los actores implicados en el territorio y lugar de análisis. La ampliación e incorporación de nuevos informantes durante la investigación, estuvo sostenida mediante la aplicación de la técnica Bola de Nieve (Martínez Salgado, 2012) buscando siempre la mayor heterogeneidad entre la muestra poblacional de estudio. La información recabada de las entrevistas, fueron procesadas, sistematizadas y analizadas a partir de transcripciones escritas y sonoras mediante la utilización del software Atlas.ti, que posibilitó codificar datos y trazar variables con los resultados obtenidos; lo cual resultó de importancia, ya que a partir de esta información se logró identificar desde las perspectivas de los sujetos y sus testimonios, los sentidos de pertenencia y prácticas que realizan de manera cotidiana sobre el espacio.

El trabajo en el terreno y la observación participante estuvo enfocado a identificar las prácticas sociales territorializadas y tomar registro de los espacios específicos identificados como lugares; complementadas y refutadas por los testimonios orales que resultaron de las entrevistas. Posteriormente, con la información recopilada se elaboró una base cartográfica, a modo de materializar las prácticas sociales que dan cuenta de la reconstrucción de los territorios y lugares.

MIGRACIONES EN LA CONFIGURACIÓN TERRITORIAL DEL ÁREA DE ESTUDIO

La historia de Pedro Luro ${ }^{6}$ y de su población tiene una estrecha relación con la tradición agrícola y migrante, favorecida por las condiciones de riego en el valle inferior del río Colorado, lugar donde se emplaza la localidad. Su configuración territorial inicial tuvo que ver con el corrimiento de la población originaria de tipo tehuelche, luego mapuche y la colonización con el

6 Fundada oficialmente en el año 1913, con la llegada del ferrocarril. 
asentamiento de población fundamentalmente de origen europea, a través de las colonias y haciendas, a mediados y fines del siglo XIX (Casa de la Cultura, 2005; Bortolotti, 1936). Posterior a esta primera etapa de migración europea, tiene lugar un proceso de poblamiento de origen limítrofe (fundamentalmente de origen chileno) por las décadas de 1930, 1940 y 19507. Al mismo tiempo, pero precisamente a fines de la década de 1960 y a partir de la de 1970, se da otro proceso importante de migración de origen boliviana. A partir del año 2001, pero en menores proporciones que las migraciones precedentes, se inicia una migración de origen paraguaya (Torrez Gallardo, 2011). Todas estas migraciones históricas de la localidad de Pedro Luro estuvieron acompañadas no solo de factores económicos, laborales y sociales que motivaron estas movilidades, sino también de significativas consecuencias en la configuración del espacio urbano y rural.

El factor común denominador a estas migraciones fueron las inserciones laborales que posibilitaron principalmente los circuitos productivos de la cebolla. A partir de la década de 1970 se incrementó la producción de esta hortaliza dotando de una importante especialización para la región del valle inferior del río Colorado; siendo hoy la principal zona productora y exportadora de cebolla en fresco del país. De forma paralela a este crecimiento y desarrollo hortícola, un nuevo flujo migratorio se afirma en la región y corresponde a los migrantes de origen boliviano y del noroeste argentino que imponen progresivamente una nueva dinámica demográfica, cultural, territorial y económica (Torrez Gallardo y Bustos Cara, 2015). El crecimiento socio-económico, el mejoramiento en la calidad de vida, y con la creciente especialización productiva en la cebolla, se dinamizó la radicación de muchas fa-

7 Se reconoce que a partir de la década de 1930 la migración de población de países limítrofes hacia la Argentina constituyó una respuesta frente a la escasez de mano de obra en el sector primario de las economías fronterizas. Los trabajadores de estos países fueron atraídos por las ocupaciones temporarias existentes en las distintas regiones de la Argentina (Benencia, Quaranta y Souza Casadinho, 2009). milias bolivianas que llegaron entre las décadas de 1960 y 1990 a la zona de Pedro Luro. Massey (2017) establece que el asentamiento de algunos migrantes en la sociedad receptora puede resultar inevitable, aunque al inicio haya sido de carácter temporal. Actualmente esta pequeña ciudad ha percibido un crecimiento demográfico en ascenso, particularmente asociado a las migraciones. La ciudad sigue siendo destino de trabajadores temporarios de origen boliviano, paraguayo, norteño y de otras regiones del país, que en su mayoría llegan para los meses estivales de la zafra, generando una interesante dinámica de flujos para toda la región.

\section{EL BARRIO COMO CONSTRUCCIÓN TERRITORIAL}

Pedro Luro ha adoptado una configuración espacial particular, en un principio respondiendo al crecimiento espontáneo y no planificado de los asentamientos residenciales; y posteriormente asociado a la radicación de familias migrantes, que por diferentes motivos (disponibilidad de tierras, bajos costos, solidaridad o redes sociales familiares previas) se asentaron en áreas periféricas de la ciudad y en barrios de poca densidad poblacional, redefiniendo nuevos territorios barriales con identidad migrante. En la Figura 2 se puede observar la constitución de los barrios que actualmente poseen una mayor presencia de familias migrantes ${ }^{8}$. Particularmente entre el barrio Bonacina y el barrio Padre Pablo, se ha generado una dinámica singular, visibilizada en el paisaje cultural por su constitución étnica y sentido de apropiación para muchas familias migrantes de origen boliviano que allí residen. En estos territorios barriales se generan una serie de prácticas espaciales y relaciones sociales que dan cuenta de un sentido de pertenencia, de identidad y de los imaginarios colectivos que tienen los bolivianos como comunidad arraigada. 'El barrio detrás de las vías', 'el barrio de los bolivianos', 'el barrio de la feria', son algunos

8 La elaboración cartográfica del plano fue resultado del análisis histórico con la observación participante. Y se contrastó con datos sobre población migrante boliviana que habitan por radios censales, obtenidos de la Base de datos REDATAM (INDEC, 2010). 
de los nombres que identifican a este territorio los mismos habitantes y los 'otros' vecinos de la ciudad. Cabe aclarar que no quedan claros los límites entre el barrio Bonacina y el Padre Pablo, al menos para los propios habitantes, quienes lo definen como un mismo barrio, independientemente de los límites catastrales.

Así el barrio como espacio socialmente construido, fue creciendo con las primeras familias bolivianas ( $\sin$ excluir a otras de origen no migrante limítrofe o de origen chileno), quienes no solo empezaron a construir sus primeras viviendas, sino que generaron a través de las redes sociales, la acogida de las siguientes generaciones de migrantes (que tomaban al barrio como la primera opción de llegada, si es que su destino no era directamente el área rural). En el contexto de la dimensión del 'transnacionalismo' del proceso migratorio, se genera una red de articulaciones y relaciones sociales entre diferentes espacios geográficos, actuando como espacios receptores, emisores y espacios de tránsito. El crecimiento empezó a ser mayor cuando aumentaron los trabajadores y trabajadoras que llegaban para los meses de la zafra. Los/las cuales, ante la necesidad de rentar alguna residencia pequeña de manera temporaria, generaron una demanda y una inversión por parte de los locales de construir habitaciones precarias para tales fines. Muchas/os que temporariamente llegaba y se iban, empezaron a quedarse, no solo por razones laborales y económicas, sino porque ya no estaban solos, había algún que otro familiar que ya había estado antes, o algún que otro paisano migrante que había vivido su experiencia. La inversión en alguna vivienda propia o la adquisición de alguna parcela de campo, el incremento de capital, la conformación de una familia y un proyecto a futuro, fueron algunos de los factores decisivos de la radicación y con ello la generación de un sentido de pertenencia local que se fue acrecentando.

Figura 2. Barrios de Pedro Luro con mayor presencia migrante

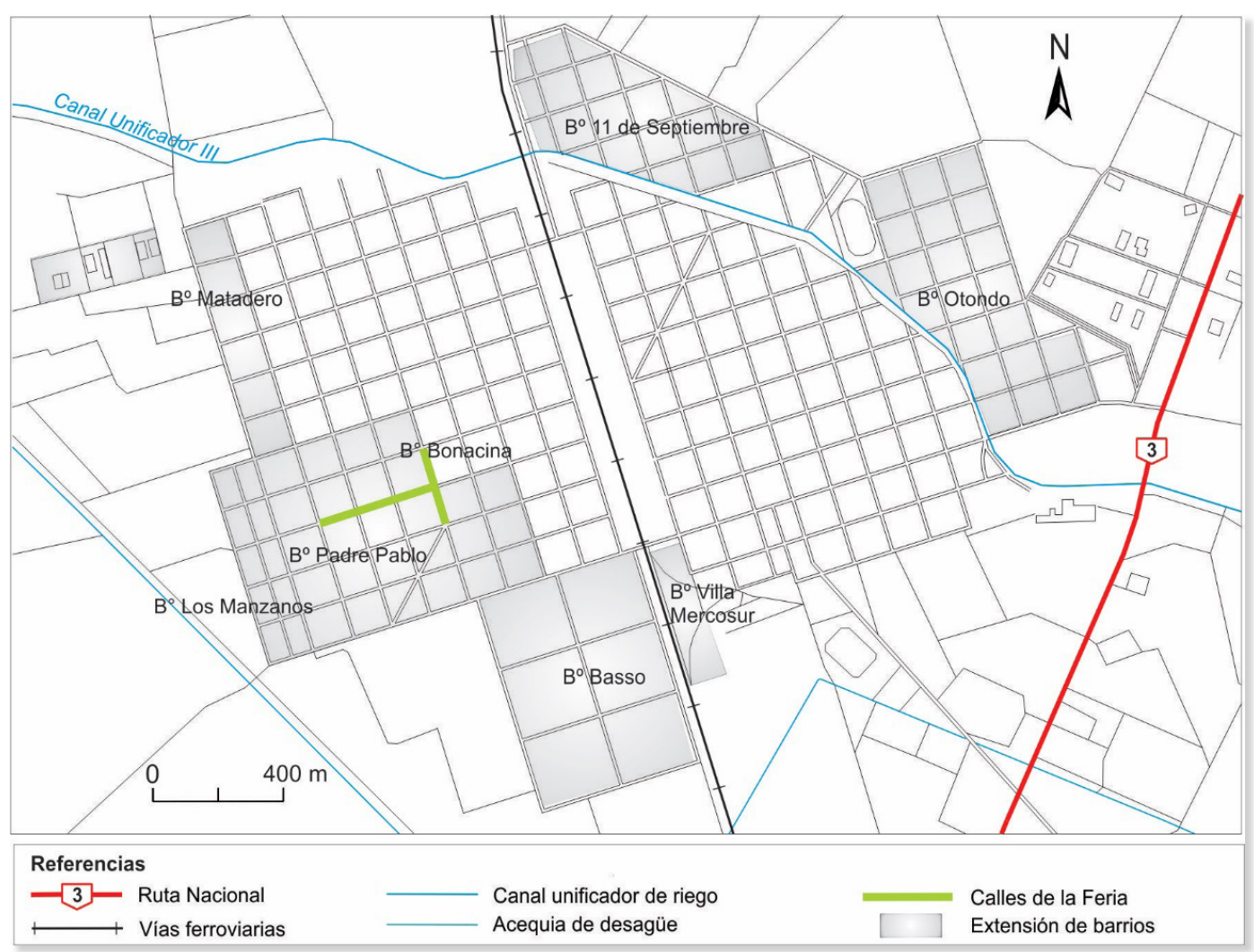

Fuente: elaboración personal, con base en el mapa catastro de la localidad de Pedro Luro 
Estébanez (1988) se refiere al barrio comunidad como aquel en el que las personas desarroIlan un sentido de colectividad y tienden a asociarse con sus vecinos más que con las personas que viven fuera del barrio, así se acentúan los patrones de segregación urbana por las fuerzas etnoculturales. Por una parte, esta idea de comunidad de una minoría étnica exige una estancia consolidada en el barrio y es consecuencia de un proceso migratorio de larga data, concretamente desde las décadas de 1980 y 1990 en adelante para el caso de estudio. Por otra parte, implica una suerte de gueto que redefine sus límites a medida que la trayectoria de sus residentes migrantes se proyecta en el tiempo, siendo inicialmente sus fronteras menos difusas y permeables. Sin embargo, estos iniciales guetos autosegregados, pueden con el tiempo ampliar sus límites territoriales y buscar intersticios o espacios de frontera para la integración social urbana, lo cual dependerá de los factores tanto geográficos como sociales del espacio, así como de las fuerzas etnoculturales de la comunidad. El 'barrio de los bolivianos', se inició como una suerte de gueto autosegregado en el área periférica de la ciudad, que con el transcurrir de los años fue adquiriendo identidad propia y ampliando sus límites territoriales, con ejemplos claros de traspasar las barreras que impone el espacio, como son las vías del ferrocarril, conscientes o no en la búsqueda de una integración social urbana, visibilizadas a través de ciertas prácticas sociales.

En el barrio aparecen prácticas espaciales y simbólicas de tipos culturales que dan cuenta de una comunidad barrial que re-construye un espacio con el que se sienten identificados o al que han dotado de ciertos elementos que, en términos subjetivos, hacen sentirlo como propio. Un ejemplo de ello son las 'prácticas religiosas' que realizan muchos bolivianos devotos de la virgen de Urkupiña. Hace unos años atrás (por el 2000 aproximadamente) integrantes de la Pastoral Boliviana lograron trasladar la imagen de la virgen a la capilla del barrio La Auxiliadora, en donde se realizan las misas dominicales y demás prác- ticas culturales asociadas a la devoción de la virgen. Los migrantes bolivianos ensayan diferentes formas de inserción y formalización de sus prácticas religiosas en los espacios urbanos (Baeza, 2006). Las fiestas populares y las danzas folklóricas, característicos de la comunidad boliviana, encuentran su seno organizativo y como punto de partida en este barrio. De algún modo estas prácticas pueden ser entendidas en el marco de las 'multicausalidades' y el 'anclaje local' de las migraciones, puesto que evidencian prácticas culturales propias y características de sus países de origen que son trasladadas y resignificadas en los nuevos espacios de los países receptores. Sassone (2007) expresa que la comunidad de migrantes bolivianos en la Argentina ha crecido en sus expresiones de religiosidad popular.

\footnotetext{
La celebración de las fiestas patronales constituye un importante elemento identitario en los barrios donde se ha rescatado esa tradición: los migrantes definen sus identidades locales en función del santo patrón y del sistema de cargos procedentes de la tradición rural y pueblerina indígena. ( $\mathrm{Li}$ socka-Jaergermann, 1998, p. 14)
}

Las prácticas religiosas pueden contribuir al fomento de la identidad étnica en el contexto migratorio, generando sentimientos muy fuertes de pertenencia a la comunidad y fomentando así la cohesión social (Sobczyk, Soriano y Caballero, 2018). La capilla ha pasado a ser el espacio de encuentro no solo de las misas, peregrinos y actividades que propone la Pastoral Migratoria, sino también como un espacio de reunión y toma de decisiones, donde los mismos vecinos se convocan para tratar distintas problemáticas que los atraviesan como comunidad. De este modo han sido posibles reuniones o asambleas para la organización de las movilizaciones por la crisis hortícola de la cebolla (conocido como el 'cebollazo') o las reuniones que llevan a cabo integrantes de la comisión de feriantes. También aparecen otros espacios que cohesionan como son las canchas de fútbol cinco, salones para eventos festivos y una amplia variedad de comercios de diversos rubros, cuyos dueños mayoritaria- 
mente son bolivianos ${ }^{9}$. Se reconfigura un territorio a partir de la apropiación de espacios públicos y privados. En las ciudades, plazas y calles, como espacios comunitarios, es donde se territorializan las prácticas del principio de vecindad, en términos relacionales.

Por otra parte, se evidencia la potencialidad de la dimensión 'subjetiva y del plano identitario' de los migrantes, que inciden y reordenan sus relaciones sociales y espaciales en su nuevo destino. En las prácticas socio-espaciales se materializan los vínculos con la tierra añorada, aparecen las emociones, sentimientos y afectividades del migrante, proyectados en las territorialidades expresadas como espacios simbólicos de memoria. El barrio aparece como un territorio donde las lógicas espaciales responden a la inversión cultural de los migrantes, como escenarios donde se materializan eventos culturales, fiestas religiosas, reuniones de los vecinos, feriantes, productores rurales y movilizaciones sociales. Es decir, se generan una serie de prácticas socio-espaciales que de alguna manera visibilizan un sentido de apropiación por parte de la comunidad boliviana. Blanco Puga y Eguren dicen que los inmigrantes en la nueva sociedad, viven dos procesos distintos, pero estrechamente relacionados:

...por un lado, los movimientos hacia la adaptación a la nueva sociedad, en la que tienen que resolver necesidades instrumentales que, en muchos casos, han sido las impulsoras de la migración, como buscar trabajo, alojamiento, escuela para sus hijos, etc. Por otro lado, los movimientos encaminados a la reconstrucción, en un nuevo y distinto contexto, de su cultura de origen, al menos en cuestiones existenciales fundamentales, como son su religión, su visión del mundo, sus pautas familiares, etc. (1996, p. 136)

9 El barrio y su comunidad ha crecido de tal manera que en los últimos años generó una demanda en términos de educación y salud. Por tanto, el municipio tuvo que gestionar unidades sanitarias con consultorios externos al hospital local, la inauguración de un nuevo jardín de infantes y la ampliación de aulas para la escuela primaria. Por otra parte, surgieron también centros comunitarios en el barrio con un grado de participación significativo de la colectividad boliviana.
LA FERIA COMO LUGAR DE IDENTIDAD Y PERTENENCIA

Según Solana, Badía, Cebollada, Ortiz y Vera (2016)

La casa, la plaza, el barrio, un pueblo, un paisaje se convierten en lugares después de un tiempo de frecuentación, de interacción e identificación con este territorio. Y el lugar forma parte constitutiva de la identidad, ya sea individual y colectiva. (p. 43)

Es la forma clave de comprender el espacio o el territorio a partir de la experiencia del sujeto o los sujetos, con toda la carga de sentido que dicha experiencia Ileva consigo. En el caso concreto del presente trabajo, es posible analizar la feria desde dos aspectos relevantes que destaca Solana et al. (2016) al referirse a la noción de lugar. Por un lado, la 'dimensión afectiva', lo que implica algún tipo de vinculación emocional para los sujetos migrantes; y por otro, los lugares tienen una 'dimensión temporal', como porciones del territorio asociadas a un pasado, a una memoria o a una historia colectiva. Por lo tanto, los lugares están cargados de significados y tienen una dimensión existencial.

Respecto a la 'dimensión temporal', la feria surge por el año 2009/2010 como consecuencia de una mala campaña de la producción de cebolla. De acuerdo a las/los migrantes entrevistadas/os, fueron algunas mujeres quienes tomaron la iniciativa de realizar ventas de empanadas, panes caseros y tamales entre los vecinos del barrio para paliar la mala racha económica de la crisis cebollera. Poco a poco estas actividades, que inicialmente complementarían la actividad laboral de la semana, empezaron a ser reiterativas de todos los domingos, sobre todo para las épocas de la zafra de cebolla, aprovechando las movilidades de los trabajadores temporarios. Tal vez tomando experiencias de otras ciudades nativas, de la capital porteña y/o de la misma Bolivia, lo que se inició de manera espontánea y ambulante, empezó a tener una mayor frecuencia haciendo uso de los mismos espacios públicos del barrio. Esta iniciativa que surge entre los vecinos del barrio, empezó a tener una mayor cantidad de adeptos (vendedores y consumidores) y a ser reconocida no solo por los residentes de la 
misma ciudad, sino también por las localidades vecinas. Al tiempo se conformó una comisión de feriantes, que organiza los puestos de ventas y ocupación del espacio público en veredas y calles del barrio (Figura 2). La ocupación de estos espacios públicos, desde una perspectiva sociocultural, pueden ser comprendidos como lugares de interrelación, de encuentro social y de intercambio (Ortiz Guitart, 2006).

Actualmente la feria se reconoce formalmente como una asociación civil, con la disposición de un estatuto que lo organiza y una comisión de feriantes miembros (mayoritariamente bolivianos). Cuenta con 200 puestos de venta aproximadamente en diversos rubros: ropa, mercadería, ropa usada, artesanías, jardinería, mercería, verduras de productores locales, especias, comidas rápidas y platos típicos de la colectividad boliviana, entre otros. Los puesteros son mayoritariamente residentes de Pedro Luro, pero también está abierta a feriantes de localidades cercanas del Partido de Villarino y de Patagones, comprendidas dentro de lo que es la zona del valle bonaerense del río Colorado. Esto último surge como acuerdo entre la comisión de la feria y el municipio, a través de la sanción de una Ordenanza 2746/2014 que reconoce a la feria como Feria Regional Barrio Bonacina; dispone de su regularización ocupando un espacio público de manera provisoria; y establece el futuro traslado de la feria a un predio cercado y acondicionado en el barrio Los Manzanos. En este sentido las interacciones sociales que se producen en la feria dan cuenta de un proceso que va cambiando con el tiempo, generando una mezcla distinta de relaciones sociales más amplias y más locales, que deriva en la especificidad del lugar (Massey, 2012).

Izquierdo Escribano y Noya (1999) en su trabajo sobre los lugares migratorios, hacen referencia citando a Giddens sobre el énfasis en la acción social situada de los migrantes y, en la importancia de analizarla desde una perspectiva que la localice en su contexto espacio-temporal. En este sentido los migrantes y los no-migrantes que concurren a la feria despliegan una serie de prácticas socio-espaciales que suceden en un día y tiempo determinado. Todos los domingos por la mañana, se pone en funcionamiento la feria barrial, convocando en promedio a cinco mil personas por día dependiendo de la estación del año y de la época de la zafra, donde confluyen vendedores, consumidores, vecinos, residentes locales y regionales. Durante este momento situado, el lugar de la feria se muestra como una configuración socio-espacial de tipo 'efímera', en el sentido de que solo sucede en un momento determinado; y en este lapso matutino el espacio adquiere significado en la misma movilidad (Hiernaux, 2006). Su carácter de efímero, posibilitaría entender no solo la disposición de los elementos materiales en un momento dado en el espacio, así como sus relaciones con otros sujetos, sino también las percepciones y las vivencias mismas de este espacio para quienes presencian o participan de los lugares efímeros. Pero la feria también configura un 'escenario' (en tiempo y espacio) que son definidos por las prácticas sociales espacializadas, y por lo tanto no existen con anterioridad a las prácticas mismas. "Un escenario es algo más que un espacio móvil en el que se despliega una práctica. Es el espacio de un conjunto de prácticas móviles y concertadas por distintos sujetos y un marco en el que toman sentido" (Lindón, 2006, p. 431).

Comprender esas percepciones, vivencias y representaciones de los sujetos, implica analizar el lugar desde una 'dimensión afectiva'. "Un lugar implica algún tipo de vinculación emocional (que puede ser el amor, pero también el odio) por parte de los seres humanos" (Solana et al., 2016, p. 43). Desde esta perspectiva interesa el 'sentido de pertenencia'10, teniendo en cuenta los sentimientos, emociones, memoria que ligan las personas a unas comunidades e identidades territoriales. En este sentido y sobre la base analítica de las entrevistas realizadas, hay opiniones encontradas de la sociedad lurense respecto a la

10 Ortiz Guitart (2006) hace referencia al sentido de pertenencia como el conjunto de sentimientos, percepciones, deseos, necesidades, construido sobre la base de las prácticas y actividades cotidianas desarrolladas en los espacios cotidianos. 
feria. Algunos la consideran como una forma de competencia comercial desigual, por la falta de pagos impositivos, por la informalidad; y otros lo encuentran como el lugar donde no solo se puede ir de compras y comercializar a precios más bajos, sino utilizarlo como un espacio para sociabilizar. Lo importante de todo esto es conocer cuál es la perspectiva de los propios vecinos del barrio, como viven la feria y su dinámica. A continuación, se citan algunos testimonios de los residentes que expresan sus referencias respecto a la feria: “...para mí la feria es un medio de salvación (...) nosotros como comunidad feriante, nos cuidamos" (Amanda, hija de migrante boliviano, vecina del barrio y comerciante). "La feria no se cierra a nadie... es un medio de salvación... esto creció por la necesidad (...) al no generar puestos de trabajo, la feria va a seguir creciendo" (Gustavo, migrante boliviano y vecino del barrio). "Es bien para la comunidad, especialmente para Luro y para todos quienes quieran venir a consumir y así mismo para todos que vienen a los puestos y es bueno para todo el partido de Villarino" (Oscar, migrante boliviano, miembro de la comisión y feriante).

La feria ha pasado a constituir más que un espacio de actividad complementaria para los vecinos, ya que ha impulsado una importante demanda productiva de hortalizas para la venta en fresco, importante para los pequeños productores familiares, que se dedican a la tierra. "Es mucho más factible para el consumidor, por precios bajos, las verduras vienen directo de la chacra a la mano del consumidor" (Pedro, migrante boliviano y feriante).

\footnotetext{
...y aparte tenés una entradita por ejemplo una como mamá que hace empanadas para vender vos sabes que hiciste y gastaste una cantidad y vos sabes que la recuperas y vas a tener para pagar la luz en el momento. Eso también es importante, no es lo mismo que ponerte a sembrar y esperar a cosechar, esto es distinto sobre todo para las mujeres que hacen labores con la comida, es beneficioso porque se recupera la plata al otro día. (Sandra, hija de migrante boliviano y vecina del barrio)
}

Sandra menciona además la importancia que tiene para ella realizar actividades económicas relativamente seguras, dando cuenta de las impredecibilidades de la producción cebollera, a la cual se dedica mayoritariamente la población boliviana. La feria adquiere una dinámica identitaria en varios sentidos, no solo por cómo se originó, por el lugar donde se desarrolla, sino también por los sabores que se encuentran. La gastronomía es una de las maneras de mantener un vínculo con la tierra añorada. Las comidas típicas hacen parte de la identidad étnica ${ }^{11}$. En contextos migratorios como el caso de Pedro Luro, donde la etnicidad se re-inventa, los referentes culturales se amplían en procesos específicos. El lugar de la feria es un lugar donde se pueden degustar comidas típicas bolivianas en algunos puestos; encontrar insumos necesarios para la elaboración de platos; y elementos prácticos para la realización de rituales culturales. Muchos de estos productos (importados desde Bolivia o de ciudades grandes como Buenos Aires) son muy valorados por parte de la comunidad boliviana, ya que forman parte de la dieta alimentaria o de las prácticas culturales que se llevan a cabo para ciertas fechas conmemorativas. Entrena Durán (2012) citando a García Canclini, menciona que la experiencia de los inmigrantes implica procesos específicos a través de los cuales se conservan, reintroducen y recrean ritualmente signos de su identidad nacional, costumbres, festivales o celebraciones religiosas. Dichos procesos, que acontecen de manera singular en cada caso, manifiestan $y$ reproducen las diferenciaciones existentes $u$ originan nuevas diferenciaciones entre distintos grupos sociales de inmigrantes.

\section{¿LA FERIA BARRIAL COMO UN ESPACIO DE INTEGRACIÓN?}

Pero la feria y el barrio no son solo lugares significativos y cargados de emotividad y sentido de pertenencia para la comunidad que allí reside, mayoritariamente boliviana; también se ha convertido en un lugar de encuentro y relaciones

11 La identidad étnica es un tipo específico de identidad social, que no excluye otras identificaciones, pero que supone la necesidad de comprenderla en todas las dimensiones que le otorgan su singularidad y la distinguen de otras identidades posibles, sin olvidar que no es esencial, sino que depende de los contextos interactivos; es decir de la confrontación con otras identidades (Bartolomé, 2006, p. 39). 
sociales con otros migrantes, muy visibilizados para los meses de la zafra, donde el paisaje cultural se torna de una mayor diversidad étnica. Es posible encontrarse con trabajadoras/es paraguayas/os, santiagueñas/os, misioneras/os, jujeñas/os e incluso brasileñas/os; que hallan en la feria el espacio para el encuentro y el intercambio después de una intensa semana laboral. Estas prácticas socio-espaciales dieron posibilidad a generar un espacio de intercambio y puesta en valor de un territorio barrial, que por lo general ha estado estigmatizado por los criollos del pueblo por ser el 'barrio de los bolivianos' (desde una connotación segregativa). No quiere decir que haya dejado de serlo, sino que hoy la feria del barrio puede ser pensada como el lugar que integra (aunque sea solo por unas horas $y$ desde el carácter efímero del espacio), en el que confluyen vecinos de distintas partes de la ciudad, de distintas comunidades étnicas y de las localidades vecinas:

Yo como mujer lo veo lindo para pasear, venir con la fami-
lia, porque es tranquilo, no hay peligro, esto trae mucha
gente. Viene gente de Conesa de Origones, de todos los
pueblos, vienen todos y muchas veces pasa de que no se
ven mucho tiempo y se ven acá. Y eso está bueno. (Veci-
na de barrio e hija de migrantes europeos)

De alguna manera se pone en evidencia el carácter 'multifacético' de las migraciones; desde la perspectiva constructiva de los imaginarios, los estereotipos económicos, sociales, entre otros; por los múltiples efectos que generan tanto en el país de origen como en el de destino. Finalmente se cita a continuación otro de los testimonios, en este caso perteneciente a una vecina del barrio de origen migrante, que da cuenta de lo que implica para ella y su familia haber tomado la decisión de establecerse en la ciudad y llevar adelante un proyecto de vida:

\begin{abstract}
No nos iríamos de Luro, tuvimos muchas ofertas, pero nos decidimos quedarnos e invertir más en Luro. Conseguimos otro local y estamos bien, vivimos bien, no nos falta nada. Hicimos este local, las piezas, no me quejo. Salimos todos los domingos a la feria, todas las mañanas salimos a la feria, vendemos bien en la feria, esto es nuestro. (Beatriz, hija de migrante boliviana y vecina del barrio)
\end{abstract}

Esta última pone en evidencia un sentido de pertenencia al territorio barrial y expresa un valor simbólico que se le atribuye al lugar de la feria; que emana como un espacio que se fue reconstruyendo en el tiempo, dinamizado por los mismos vecinos, que étnicamente le otorgaron una identidad colectiva, y que empieza a ser reconocida por el resto de los residentes locales. Las actividades comerciales motivan ciertas lógicas de interacción, que exceden las finalidades puramente comerciales, pudiendo generar mecanismos de relaciones sociales que permean las fronteras de pertenencia étnica, dando lugar a un espacio de interacción e integración social.

\section{REFLEXIONES FINALES}

Los migrantes bolivianos en Pedro Luro, quienes poseen una significativa trayectoria para la región, han puesto en evidencia un sentido de pertenencia y de apropiación colectiva, a partir de diferentes prácticas socio-espaciales desplegadas tanto en el barrio que los identifica, como en la organización de la feria. El barrio de los bolivianos y la feria como unidades de análisis, demuestran las cargas simbólicas que implican estos espacios para los sujetos de la comunidad migrante, en donde ellos tienen una participación muy activa. Los procesos identitarios e imaginarios colectivos se plasman a través de las prácticas sociales (como las religiosas en torno a la virgen de Urkupiña, las culturales como las danzas folklóricas y las comerciales de la feria) que llevan a cabo de manera compartida como comunidad sobre un espacio geográfico determinado. El barrio como territorio y la feria como lugar, aparecen como construcciones situadas por los migrantes bolivianos, con un claro sentido de pertenencia que se proyecta y se acentúa en el tiempo. En este sentido el espacio deja de ser una extensión neutra, en términos de Claval (2002), sino una escena donde los actores se dejan ver; el espacio se asemeja a un texto, puesto que está cargado de mensajes que le confieren un sentido. Las percepciones de los sujetos y sus improntas en el discurso y en el mismo paisaje dan cuenta de esos sentidos. "Quienes lo modelan, intentan plasmar en la realidad sus perspec- 
tivas, sus sueños y esperanzas. También intentan justificar su postura dominante, a través de la creación de paisajes (...)" (Claval, 2002, p. 32).

Es en ese paisaje y en las mismas prácticas sociales espacializadas, donde los sujetos migrantes conciben sus historias, proyectos de vida, tradiciones y memorias colectivas de un origen lejano; de allí que resulta importante considerar las multidimensionalidades de lo que implica el proceso migratorio como proceso social. No solo se trata de un fenómeno de movilidad demográfica con un anclaje territorial en un espacio receptor; sino que existe toda una complejidad que atraviesa al migrante como sujeto social de tiempo y espacio, desde el momento en que decide salir de su país hasta el momento en el que decide quedarse y proyectar un futuro sobre un espacio en el que le fue indiferente en un principio. Existe una carga subjetiva e identitaria que acompaña al migrante boliviano y despliega en su proyecto de reterritorialización en el nuevo espacio, donde interacciona en mayor o menor medida con las dimensiones subjetivas e identitarias del residente local, generando límites más o menos permeables con el territorio barrial que se reconstruye. El carácter multifacético del proceso social de la migración, así como el anclaje local y el transnacionalismo, aparecen como dimensiones que permitieron comprender la lógica del proceso social migratorio boliviano en Pedro Luro, contextualizado en un proceso mayor donde tienen lugar estos fenómenos, excediendo los límites de la escala local.

De acuerdo a las entrevistas realizadas y a las prácticas identificadas, se encontraron heterogeneidades entre los actores bolivianos y los otros migrantes, puesto que los segundos no han tenido el nivel de difusión y materialización

\section{REFERENCIAS}

Baeza, B. (2006). Chilenos y bolivianos en Comodoro Rivadavia (Chubut) (pp. 353-378). En A. Grimson y E. Jelin (Comps.) Migraciones regionales hacia la Argentina. Diferencia, desigualdad $y$ derechos. Buenos Aires: Prometeo. en el espacio en comparación con los primeros. Por tanto, no han adquirido un nivel de relevancia que se visibilice en el espacio y sea reconocido por los 'otros' residentes de la ciudad. Para el caso de la migración boliviana, existe un claro reconocimiento por parte de los otros colectivos y de la población local 'criolla', en su adscripción espacial y socio-cultural, lo que surge como parte de los testimonios registrados y la observación participante. Claramente esto no solo tiene que ver con la trayectoria de los colectivos migrantes presentes en la localidad, la cantidad en su conjunto, sino también con las características étnicas que componen a cada grupo social. Por su parte, se percibe que las generaciones de nacidos en Argentina han sido parte fundamental de ese sentido de pertenencia y de apropiación territorial, puesto que representan a los proyectos de vida en la radicación definitiva de las primeras familias bolivianas. Por otra parte, han sido y son actores clave en la reafirmación y resignificación de la bolivianidad como colectividad, expresadas en su participación activa en torno a las diversas actividades que desarrollan como grupo social.

Analizar casos de estudios vinculados a los procesos sociales que contribuyan a comprender las perspectivas, sentimientos, percepciones e imaginarios de los sujetos, implica una complejidad en sí misma, por la cual la inmersión en el plano metodológico se hace desde un enfoque cualitativo. De allí las potencialidades del enfoque disciplinar de la Geografía Cultural, como ciencia social, puesto que la misma posibilita cambiar la perspectiva analítica para comprender y pensar al sujeto en su relación intrínseca con los otros, desde una dimensión colectiva y contextuada.

Bartolomé, M. (2006). Los laberintos de la identidad. Procesos identitarios en las poblaciones indígenas. Avá, (9), 28-48.

Bauman, Z. (1994). Pensando sociológicamente. Buenos Aires: Nueva visión. 
Benencia, R. (2006). Bolivianización de la horticultura en la Argentina. Procesos de migración transnacional y construcción de territorios productivos (pp. 135-167). En A. Grimson y E. Jelin (Comps.) Migraciones internacionales en la Argentina. Diferencia, desigualdad y derechos. Buenos Aires: Prometeo.

Benencia, R. (2007). Bolivianización de la horticultura en la Argentina (pp. 135-167). En A. Grimson y E. Jelin (Comps.) Migraciones regionales hacia la Argentina. Diferencia, desigualdad y derechos. Buenos Aires: Prometeo.

Benencia, R.; Quaranta, G. y Souza Casadinho, J. (2009). Cinturón hortícola de la Ciudad de Buenos Aires. Cambios sociales y productivos. Buenos Aires: CICCUS.

Blanco Puga, M. y Eguren, J. (1996). La ayuda mutua como acción intercultural. Migraciones, 135-145.

Bortolotti, E. (1936). Pedro Luro y las estaciones del ferrocarril a Patagonia. Diario El Atlántico, 9 de julio. Bahía Blanca.

Bustos Cara, R. (2002). Cambios en los sistemas territoriales. Actores y sujetos entre la estructura y la acción. Propuesta teórica-metodológica. II Jornadas Interdisciplinarias del Sudoeste Bonaerense. Bahía Blanca: EdiUNS.

Carreras, C. y García Ballesteros, A. (2006). La Geografía Urbana (pp. 84-94). En A. Lindon y D. Hiernaux (Dirs.) Tratado de Geografía Humana. México: Anthropos.

Casa de la Cultura. (2005). Vivencias de Ayer y de Hoy. Tomo I. Pedro Luro: Casa de la Cultura.

Chmiel, S. (1999). Los otros que son nosotros. Diáspora y construcción de identidades. En M. Margulis y M. Urresti La segregación negada. Cultura y discriminación social. Buenos Aires: Biblos.

Claval, P. (2002). El enfoque cultural y las concepciones geográficas del espacio. Boletín de la Asociación de Geógrafos Españoles, (34), 21-39.

Clua, A. y Zusman, P. (2002). Más que palabras: otros mundos. Por una geografía cultural crítica. Boletín de la Asociación de Geógrafos Españoles, (34), 105-117.

Cortès, G. (2009). Migraciones, construcciones transnacionales y prácticas de circulación. Un enfoque desde el territorio. Párrafos Geográfi$\cos , 8(1), 35-53$.

Devoto, F. (2003). Historia de la inmigración en Argentina. Buenos Aires: Sudamericana.

Entrena Durán, F. (2012). Migraciones globales y reterritorialización de los espacios locales: una aproximación tridimensional. Papeles de Población, 72.

Estébanez, J. (1988). Los espacios urbanos. En R. Puyol, J. Estébanez y R. Méndez Geografía Humana. Madrid: Cátedra.

Feldman-Bianco, B.; Rivera Sánchez, L.; Stefoni, C. y Villa Marínez, M. (2011). La construcción social del sujeto migrante en América Latina: prácticas, representaciones y categorías. Quito: FLACSO.

Fernández Christlieb, F. (2006). Geografía cultural. En D. Hiernaux y A. Lindón (Dirs.) Tratado de geografía humana. México: Anthropos.

Garín Contreras, A. (2013). Pobreza y Segregación Socioespacial en una ciudad intermedia: el caso de Temuco 1990-2013. Tesis de doctorado en Geografía. Bahía Blanca: Universidad Nacional del Sur. Grimson, A. y Jelin, E. (2006). Migraciones regionales hacia la Argentina. Diferencia, desigualdad y derechos. Buenos Aires: Prometeo.

Halpern, G. (2009). Etnicidad, inmigración y política. Representaciones y cultura política de exiliados paraguayos en Argentina. Buenos Aires: Prometeo.

Hiernaux, D. (2006). Geografía de los tiempos y de los espacios efímeros y fugaces (pp. 269284). En J. Nogué y J. Romero (Eds.) Las otras geografías. Barcelona: Tirant lo Blanch.

Instituto Nacional de Estadística y Censos (INDEC) (2010). Base de datos REDATAM. Censo Nacional de Población, Hogares y Viviendas 2010. Recuperado de https://www.indec.gob.ar/indec/web/Institucional-Indec-BasesDeDatos-6 (consulta marzo de 2020).

Instituto Nacional de Estadística y Censos (INDEC) (2015). Estimaciones de población por sexo, departamento y año calendario 2010-2025. Buenos Aires: INDEC.

Izquierdo Escribano, A. y Noya, J. (1999). Lugares migratorios. Una propuesta teórica y me- 
todológica para el análisis de la integración social de los inmigrantes. Migraciones, 6, 19-42.

Lamborghini, E. y Martino, M. (2018). La dimensión global de las migraciones actuales. Buenos Aires, Argentina: Facultad Latinoamericana de Ciencias Sociales.

Lara Flores, S. (2012). Los territorios migratorios como espacios de articulación de migraciones nacionales e internacionales. Cuatro casos del contexto mexicano. Política y Sociedad, 49(1), 89-102.

Lindón, A. (2006). La espacialidad de la vida cotidiana: hologramas socioterritoriales de la cotidianeidad urbana (pp. 425-445). En J. Nogué y J. Romero (Eds.) Las otras geografías. Barcelona: Tirant lo Blanch.

Lindón, A. (2007). El constructivismo geográfico y las aproximaciones cualitativas. Norte Grande, 37, 5-21.

Lins Ribeiro, G. (2012). La globalización populary el sistema mundial no-hegemónico. Nueva Sociedad, 36-62.

Lisocka-Jaergermann, B. (1998). Los espacios étnicos en las grandes metrópolis: ¿Pueblos en las urbes? (pp. 13-19). En B. Lisocka-Jaergermann (Ed.) El espacio en la cultura latinoamericana 4. Memorias de la III Reunión del Proyecto (Quito 7-11 de julio), Varsovia, Universidad de Varsovia, Centro de Estudios Latinoamericanos.

Marshall, C. y Rossman, G. (1995). Designing Qualitative Research. California: Sage.

Martínez Salgado, C. (2012). El muestreo en investigación cualitativa. Principios básicos y algunas controversias. Ciência \& Saúde Coletiva, 12(3), 613-619.

Massey, D. (2012). Un sentido global del lugar. En A. Albet y N. Benach. (Eds.) Doren Massey, un sentido global del lugar. Barcelona: Icaria.

Massey, D. (2017). Comprender las migraciones internacionales (pp. 112-129). Teorías, prácticas y políticas migratorias. Barcelona: Edicions Bellatera. Municipalidad de Villarino (2014). Ordenanza $N^{\circ}$ 2746.

Novick, S. (2008). Las migraciones en América Latina. Buenos Aires: CLACSO.
Organización Internacional de las Migraciones (OIM) (2006). Glosario sobre Migración, Derecho internacional sobre Migración, (7). Recuperado de http://publications.iom.int/system/files/pdf/ iml_7_sp.pdf

Ortiz Guitart, A. (2006). Usos de los espacios públicos y construcción del sentido de pertenencia de sus habitantes en Barcelona (pp. 67-83). En A. Lindón, M. Aguilar y M. Hiernaux (Coords.) Lugares e imaginarios en la metrópolis. México: Anthropos.

Pacecca, M. (2009). La migración boliviana, peruana y paraguaya a la Argentina (1980-2005). Congress of the Latin American Studies Association. Rio de Janeiro, Brasil.

Pizarro, C. (2011). Migraciones internacionales contemporáneas. Estudios para el debate. Buenos Aires: Ciccus.

Sassen, S. (2006). Inmigrantes en la Ciudad Global. Recuperado de http://www.pvp.org.uy/saskia. htm (consulta 12 de enero de 2019).

Sassone, S. (2007). Migración, territorio e identidad cultural: construcción de lugares bolivianos en la Ciudad de Buenos Aires. Población de Buenos Aires, 4(6), 9-28.

Sassone, S. y Hughes, J. (2009). Fe, devoción y espacio público: cuando los migrantes construyen lugares (pp. 151-174). En C. Carballo (Coord.) Cultura, territorios y prácticas religiosas. Buenos Aires: Prometeo.

Sassone, S. y Mera, C. (2007). Barrios de migrantes en Buenos Aires: Identidad, cultura y cohesión socioterritorial. Preactas V Congreso Europeo CEISAL de latinoamericanistas -Consejo Europeo de Investigaciones Sociales de América Latina- Las relaciones triangulares entre Europa y las Américas en el siglo XXI: expectativas y desafíos. Recuperado de https://docplayer.es/4610731-Barrios-de-migrantes-en-buenos-aires-identidad-cultura-y-cohesion-socioterritorial.html (consulta 12 de enero de 2019).

Sauer, C. (1995). La morfología del paisaje (pp. 91-96). En J. Bosque Maurel y F. Ortega Alva Comentario de textos geográficos (historia y crítica del pensamiento geográfico). Barcelona: Oikos-Tau. 
Scoot, A. y Soja, E. (1996). The city. Los Angeles and Urban theory at the end of the twentieth century. Berkeley, Los Angeles: University of California Pres.

Scribano, A. (2008). El proceso de investigación social cualitativo. Buenos Aires: Prometeo.

Sobczyk, R.; Soriano, R. y Caballero, A. (2018). El mercado laboral y la religión: la religión "vivida" de la diáspora comercial de Otavalo (Ecuador). Migraciones, 45, 3-28. DOI: mig. i45y2018.001

Soja, E. (2000). Postmetropolis. Oxword: Blackwell. Solana, M.; Badía, A.; Cebollada, Á.; Ortiz, A. y Vera, A. (2016). Espacios globales y lugares próximos. Setenta conceptos para entender la organización territorial del capitalismo global. Barcelona: Icaria.

Tarrius, A. (2009). Intérêt et faisabilité de l'approche des territoires des circulations transnationales (pp. 43-51). En G. Cortès y Faret, L. Les circulations transnationales. Lire les turbulences migratoires contemporaines. Paris: Armand Colin.

Torres, F. (2011). Territorio y lugar: potenciali- dades para el análisis de la constitución de los sujetos políticos. El caso de un movimiento de desocupados en Argentina. Geograficando, 7(7), 209-238.

Torrez Gallardo, M. (2011). Transformaciones socio-espaciales en Pedro Luro, vinculadas a las migraciones de las últimas décadas. Partido de Villarino. Párrafos Geográficos, 10(1), 570-592.

Torrez Gallardo, M. y Bustos Cara, R. (2015). Las dinámicas migratorias en la región. El caso del sudoeste de la provincia de Buenos Aires (pp. 171-177). En M. Sili La región del Colorado: historia, cultura y paisaje en la frontera. Ciudad Autónoma de Buenos Aires: el autor.

Vasilachis de Gialdino, I. (2006). Estrategias de investigación cualitativa. Barcelona: Gedisa.

Wagner, P. (2002). Cultura y geografía: un ensayo reflexivo. Boletín de la Asociación de Geógrafos Españoles, (34), 41-50.

Wihtol de Wenden, C. (2013). El fenómeno migratorio en el siglo XXI. Migrantes, refugiados y relaciones internacionales. México D.F: Fondo de Cultura Económica.

\begin{abstract}
Marcela Torrez Gallardo es Licenciada y Profesora en Geografía por la Universidad Nacional del Sur (UNS). Diplomada Superior en Migraciones, Movilidades e Interculturalidad en América Latina por FLACSO. Actualmente es becaria doctoral en Geografía por el CONICET. Docente en el nivel Superior Terciario (I.S.F.D. $N^{\circ} 3$ ) y Universitario (UNS). Investigadora del Colectivo de Estudios e Investigaciones Sociales y miembro del GT Pensamiento Geográfico Crítico Latinoamericano de CLACSO. Desarrolla investigaciones asociadas a las transformaciones territoriales y a los estudios migratorios, desde la perspectiva de la integración y la segregación socio-espacial; con un enfoque cultural en las lógicas de las movilidades sociales y la apropiación del espacio. Ha participado de diversos seminarios y encuentros académicos a nivel nacional e internacional; con la publicación en revistas, libros y actas con y sin referato. Departamento de Geografía y Turismo. Universidad Nacional del Sur. 12 de Octubre y San Juan, 4 Piso, Gabinete 12, (8000) Bahía Blanca, Buenos Aires, Argentina, torrez_marcela@hotmail.com
\end{abstract}

